\title{
Is the difference between gill and girl more than a letter?
}

\author{
GREGG C. ODEN and JAY G. RUECKL \\ University of Wisconsin, Madison, Wisconsin
}

\begin{abstract}
Letter-like characters from continua ranging between $t$ and $l, d$ and $l$, or $i$ and $l$ were factorially embedded in word frames either having or not having an $l$ in a position adjacent to the variable character. In the former but not in the latter case, the letter continuum manipulation varied the degree of presence of doubled-letter parallelism as well as the degree to which the character was one letter rather than the alternative. Letter-identification rating data suggested that the parallelism property played a minor part in the process of identifying the words. This result, as well as the more general issues associated with it, are examined within the framework of a fuzzy propositional model of pattern identification.
\end{abstract}

A pattern is a particular arrangement of parts. Without the parts, there would be nothing to arrange, but without the arrangement, there would be no pattern (at least not the same pattern). Despite this seemingly obvious complementarity, models of pattern identification have tended toward extremes of emphasis on the role of parts versus the role of arrangement in making patterns recognizable. For example, there is a long history of rather intense competition between the distinctive features and holistic models of letter identification and, similarly, between the letter-by-letter and whole-word models of word identification. The former model treats patterns as being no more than disjoint collections of components, whereas the latter model makes no use of the fact that patterns can be analyzed into constituents.

An alternative to these models is to acknowledge that patterns have many different properties, some arising from characteristics of parts and some arising from how those parts are put together in the pattern (see Oden, 1984a, for additional discussion). The task then is to determine which of these properties are exploited in the process of recognizing the pattern. The present study takes this point of view in addressing the potential role of a particular property of some words that arises from the arrangement of their letters, namely, the parallelism that results from doubled letters. In particular, we examine whether the visual parallelism of doubled lowercase $l s$ independently contributes to the recognition of words having adjacent ls. Intuitively, this property of words like spill, hollow, and llama seems to be as perceptually salient as the intrinsic features of the letter $l$ itself. Indeed, it has been well established (e.g., Pomerantz \& Garner, 1973; Pomerantz, Sager, \& Stoever, 1977) that features such as parallelisms and symmetries are available to the perceptual system and, generally, can play a very important

This research was supported by NSF Grants BNS80-14316 and BNS8310870. Address correspondence to G. Oden, Department of Psychology, University of Wisconsin, Madison, WI 53706. role in visual pattern recognition. The present issue, then, is whether or not the representation and recognition of words as visual patterns make use of such features.

To address this issue, we used the continuous feature manipulation approach, which has proven useful in exploring questions such as this in the past (Massaro, 1979; Naus \& Shillman, 1976; Oden, 1979, 1984a). In the present case, three letter continua were constructed (see Figure 1), ranging in small steps from well-formed $t, d$, and $i$ characters to a well-formed $l$ in each instance. For each continuum, the characters were individually embedded in each of two word-context letter frames, one of which itself contained an $l$. For example, the $t / l$ series characters were combined with cul_ and cur_ frames. In the former case, the result was a word continuum ranging from cult to cull, whereas in the latter case, the continuum ranged from curt to curl. Thus, the former, but not the latter, continuum involved the absence versus
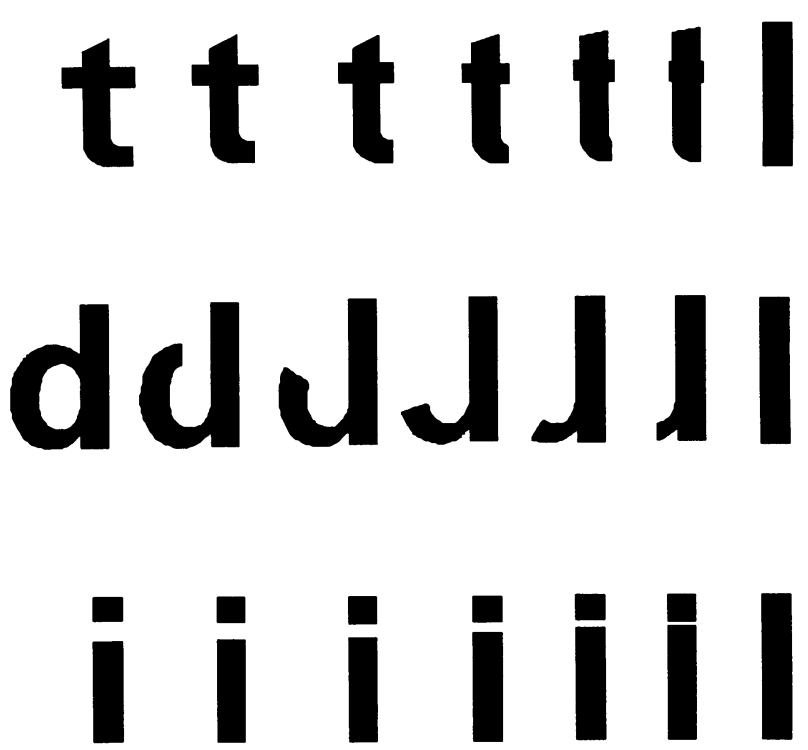

Figure 1. The $t / l, d / l$, and $i / l$ letter continuum stimulus series. 
presence of the parallelism property in addition to the degree to which the fourth letter was $t$ or $l$. The logic of this design is that if the parallelism property is functionally important, then the two frames should produce a difference in the identification of the words as a function of the $t / l$ series level. Specifically, we expected that the degree to which this property is absent from the stimulus should penalize the word cull relative to cult but not curl relative to curt. As a consequence, there would be a separation of the identification curves in the region of the letter series where the character is at least somewhat of an $l$, but for which the parallelism property is not yet perfect (i.e., near to, but not at, the $l$ end of the series).

All of this assumes, of course, that the continuous letter series manipulation is a meaningful one, that is, that the nature and degree of the deviations of the intermediate characters in Figure 1 from the canonical characters at the ends of the series are informative, and not just some sort of perceptual noise that would be immediately discarded by a peripheral clean-up operation. Similarly, the experimental logic presupposes that it makes sense that word responses could be "penalized" and not just totally eliminated by relatively minor deviations from the ideal word pattern. These presumptions are natural and, in fact, basic within the conceptual framework that we adopt, that of the fuzzy propositional model of pattern identification (Oden, 1979; Oden \& Massaro, 1978). In this model, patterns are represented internally by propositions whose elementary terms are fuzzy predicates-predicates that may be more or less true of any object-and whose connectives preserve the fuzziness thus introduced, so that the entire proposition is more or less true of any stimulus as a function of the degree to which it has a proper assortment of the relevant properties. Although the present study is not designed to provide a test of this model (it has already received substantial empirical validation; e.g., Massaro, 1979; Oden, 1979, 1984a; Oden \& Massaro, 1978; Rueckl \& Oden, in press), this study does illustrate how the model provides a useful theoretical framework that can facilitate the asking of questions that are of general interest.

\section{METHOD}

Subjects used a line-mark rating scale to indicate their judgments of the degree to which each individually presented stimulus was one word relative to the alternative.

\section{Stimuli and Design}

There were three $2 \times 7$ factorial stimulus matrices, one for each of the seven-level letter series shown in Figure 1. The two-level factor with which each series was combined consisted of a pair of word frames contrasting on whether or not they included a letter $l$ : cul_ versus cur_, gil_ versus gir_, and wa_l versus wa_t for the $t / l, d / l$, and $i / l$ series, respectively. Since the word frames within each pair differed from each other in only a single letter position, an alternative way of looking at this factor is as involving a very reduced letter-series "continuum."

The stimuli were constructed from Mecanorma press-on letters in 48point Univers 83 type. Each intermediate member of each letter series was handcrafted, using an Exacto knife to cut away increasing increments of material from the larger (physically more encompassing) of the two end letters for each series, $t, d$, and $l$. To ensure uniformity across each level of each factor, the word frames were first laid out and then xeroxed seven times, and each letter series was xeroxed twice. The stimuli were then fabricated by pasting the letter series characters into the appropriate word frames. The stimuli were individually photographed using high-contrast film and were projected onto a rearprojection screen approximately $100 \mathrm{~cm}$ from the subject. The stimuli subtended from $3.5^{\circ}$ to $4^{\circ}$ horizontally.

\section{Procedure}

Each subject was run separately through a single session lasting approximately $45 \mathrm{~min}$. At the start of the session, instructions describing the task and the use of the response scale were read to the subject. Following 12 practice trials, there were five blocks of experimental trials in which the 42 stimuli were presented in random order.

On each trial, the stimulus slide was displayed on the screen for $100 \mathrm{msec}$, followed by a dark field; this should not have limited the visual information available to the subject, but did prevent prolonged study. The subject then judged the degree to which the stimulus was one alternative relative to the other by drawing a mark through an $85-\mathrm{mm}$ line in a response booklet; the position of the mark along the line represented the rating. The ends of the line were designated by the relevant response alternatives for the word frame of the stimulus (e.g., cull and $c u l t$ ), with assignment of response alternatives to the ends of the line being random. Thus, each extreme end of the line represented perfect match of the stimulus to the respective word pattern, whereas intermediate positions represented correspondingly intermediate degrees of relative match to the two alternatives.

The line-mark positions were measured by the experimenter after the session. The data means reported here have been normalized by dividing by 85 in order to yield a relative judged degree-of-match scale ranging from zero to one.

\section{Subjects}

Twenty-four University of Wisconsin undergraduate psychology students participated in the experiment in exchange for extra course credit.

\section{RESULTS}

The results are presented in Figure 2. In this figure, each panel represents the data for one of the three matrices. The data are given as the normalized mean rated degree to which each stimulus was the word with $l$ versus the word with $t, d$, or $i$ in the "blank" in the word frame. In each panel, the two curves correspond to the two word frames and are plotted as a function of the level of the $t / l, d / l$, or $i / l$ series. As is immediately apparent from the figure, all three feature manipulation factors had the expected potent effect, driving responses from one extreme to the other over the course of the series $[F(6,138)=$ $353.830,116.664$, and 251.398 , all $p s<.001$, for main effect for the three matrices, respectively]. It is equally apparent that the word context manipulation had little, if any, effect overall; only that for the second (gil_/gir_) matrix reached significance $[F(1,23)=8.150, p<.01]$. However, any effect of a doubled-letter feature would be expected to show up only for certain regions of the letter continuum factor. Therefore, we need to take a closer look at the details of the pattern of the interaction for each matrix in turn.

For the cul_/cur_matrix, the results exhibit the general pattern that would be expected if a separate parallelism feature were operative: the cul_ curve lies below the cur_ curve for two of the levels toward the $l$ end of the $t / l$ let- 

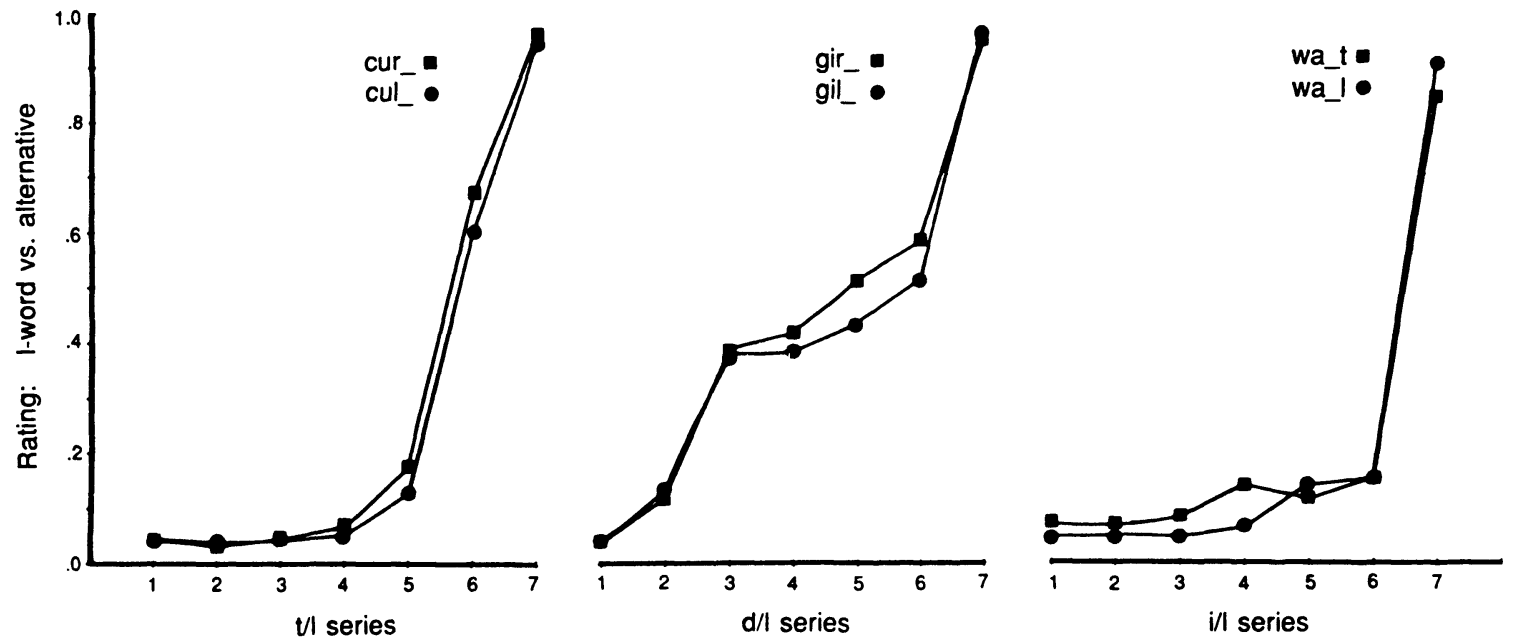

Figure 2. The three panels show the mean rated degree to which each stimulus was identified as being the word with $l$ rather than the word with $t, d$, or $i$, respectively; in each panel, the data for the two word frames are plotted as a function of the seven-level letter continuum series.

ter series. However, this effect is not statistically reliable, either as assessed by the overall interaction $[F(6,138)=$ .737], or by specific comparisons computed at levels 5 and $6[F(1,23)=2.026$ and .915 , respectively $]$.

For the gil_/gir_ matrix, the same pattern is obtained, but in this case, it is more pronounced, extending over three of the seven letter-series levels. The effect is also reliable, both as measured by the interaction $[F(6,138)$ $=3.123, p<.01]$, and by specific comparisons at level $5[F(1,23)=6.236, p<.05]$ and level $6[F(1,23)=$ $8.205, p<.01]$.

Finally, for the wa_l/wa_t matrix, although the interaction approaches significance $[F(6,138)=2.098$, $p<.10$ ], the pattern of the interaction is not that which would result from the hypothesized parallelism feature. On the other hand, the form of the two curves as a function of the $i / l$ series is such as to preclude any opportunity for the relevant pattern to be observed, whether the hypothesis is correct or not. This is because there is very little between ceiling and floor where the two curves would have a chance to separate in the systematic fashion that is required. In other words, the letter continuum factor failed to be functionally continuous as is necessary for the test of the hypothesis.

Taken together, then, the results are somewhat mixed, but contain repeated (if not universal) evidence for a weak effect exhibiting the pattern that would follow from a parallelism feature being used during the identification process.

\section{DISCUSSION}

Results such as these might be used to argue either way regarding the original experimental question. Certainly, only one of the three matrices provides solid evidence for the hypothesized use of a parallelism feature, and even for that matrix, the effect is small. On the other hand, the results are in the direction expected under the hypothesis in every place where it is possible for them to be, even though they are significantly so only in a subset of those places. All in all, it is perhaps most reasonable to conclude that a parallelism feature probably is involved, but that it plays, at most, a secondary role in identification. In fact, effects of such "supersegmental" features are notoriously elusive, and the present evidence for one is about as strong as any to be found in the word identification literature (see Oden, 1984a, for a fuller discussion).

The type of conclusion that we are advocating-that configural or emergent relational properties, such as parallelism, may play a role in word identification, but not a very important one-may be dissatisfying to those who have relished the stark contrast offered by the traditional choice between distinctive feature and holistic theories. However, we would argue not only that this conclusion is called for by the data of the present and previous studies (e.g., Oden, 1984a), but also that it more realistically reflects the character of the word as a visual entity. That configural properties become part of one's representation about the form of a word seems inevitable, given the already mentioned perceptual salience of such features and the fact that words are highly recurrent, familiar patterns. On the other hand, that such properties play only a secondary role in identifying words simply reflects the highly composite linguistic structure of words: they are made up directly of letters, and consequently, their most common and widely useful visual properties are those that belong to individual letters. Thus, our conclusion can be seen as a natural result of the principle we have repeatedly propounded (e.g., Massaro \& Oden, 1980; Oden, 1979, 1984b), that identification and comprehension processes exploit all kinds of available information, each to the degree to which it is useful.

As we observed at the beginning of this paper, the present work also highlights another way in which our approach differs importantly from both the distinctive-features and the holistic points of view. In contrast to the former, we believe that the structural, relational properties of patterns are every bit as important as the parts from which patterns are constructed (see Oden 1979; Sanocki, 1985). Therefore, provision must be made, as it is in the fuzzy propositional model, for relational features of all sorts to be included in pattern specifications along with the more traditional type of componential features. On the other hand, unlike the holistic position, our approach is based on the premise that identification (and cognition generally) is analytic (i.e., built up from separate, more elementary subunits). Again, this aspect is reflected in the fuzzy propositional model, in this case by the fact that all features, relational as well as componential, are assumed to be evaluated and processed completely independently before being integrated according to the specification of the pattern proposition. In sum, the issue addressed by the 
present study provides yet another illustration of the need to capture the structural character of patterns within analytic models of the identification process.

\section{REFERENCES}

MASSARo, D. W. (1979). Letter information and orthographic context in word perception. Journal of Experimental Psychology: Human Perception \& Performance, 5, 595-609.

MASSARo, D. W., \& OdEN, G. C. (1980). Speech perception: A framework for research and theory. In N. J. Lass (Ed.), Speech and language: Advances in basic research and practice (Vol. 3, pp. 129-165). New York: Academic Press.

Naus, M. J., \& Shillman, R. J. (1976). Why a Y is not a V: A new look at the distinctive features of letters. Journal of Experimental Psychology: Human Perception \& Performance, 2, 394-400.

ODEN, G. C. (1979). A fuzzy logical model of letter identification. Journal of Experimental Psychology: Human Perception \& Performance, 5, 336-352.

ODEN, G. C. (1984a). Dependence, independence, and emergence of word features. Journal of Experimental Psychology: Human Perception \& Performance, 10, 394-405.

ODEN, G. C. (1984b). Integration of fuzzy linguistic information in language comprehension. Fuzzy Sets \& Systems, 14, 29-41.

ODEN, G. C., \& MASSARO, D. W. (1978). Integration of featural information in speech perception. Psychological Review, 85, 172-191.

PomerantZ, J. R., \& GaRNeR, W. R. (1973). Stimulus configuration in selective attention tasks. Perception \& Psychophysics, 14, 565-569.

Pomerantz, J. R., Sager, L. C., \& Stoever, R. J. (1977). Perception of wholes and of their component parts: Some configural superiority effects. Journal of Experimental Psychology: Human Perception \& Performance, 3, 422-435.

RUECKL, J. G., \& ODEN, G. C. (in press). The integration of contextual and featural information during word identification. Journal of Memory and Language.

SANOCKI, T. (1985). Visual knowledge underlying letter perception: A font-specific approach. Unpublished doctoral dissertation, University of Wisconsin, Madison.

(Manuscript received for publication October 14, 1985.) 